\title{
ULTRAFAST DYNAMICS OF PHOTOCHROMIC COMPOUND BASED ON OXAZINE RING OPENING
}

\author{
M. Barkauskas ${ }^{\text {a }}$, V. Martynaitis ${ }^{\text {b }}$, A. Šačkus ${ }^{b}$, R. Rotomskis ${ }^{a}$, V. Sirutkaitis ${ }^{\text {a }}$, and \\ M. Vengris ${ }^{\text {a }}$ \\ ${ }^{a}$ Department of Quantum Electronics, Vilnius University, Saulètekio 10, LT-10223 Vilnius, Lithuania \\ E-mail: martynas.barkauskas@ff.vu.lt \\ ${ }^{\mathrm{b}}$ Institute of Synthetic Chemistry, Kaunas University of Technology, Radvilenu 19, LT-50254 Kaunas, Lithuania
}

Received 17 August 2008; revised 17 September 2008; accepted 18 September 2008

\begin{abstract}
We have performed a femtosecond pump-probe and nanosecond flash-photolysis measurements on a new type of photochromic molecules. These photochromes incorporate an indolo[2,1-b][1,3]benzoxazine ring system which opens upon UV light excitation and closes back within a few tens of ns. The details of ring opening and spectral properties of the photoproducts have been studied by investigating the spectral signatures of the model compounds representing separate structural parts of the photochromic switch. Comparison of the photoinduced dynamics of the model compounds and those of photochromic molecule has revealed a detailed molecular picture of the light-driven switch function.

Keywords: photochromism, pump-probe, flash-photolysis, indolo[2,1-b][1,3]benzoxazine, 4-nitrophenol, 4-nitrophenolate, $3 H$-indolium iodide, transient absorption, ring opening
\end{abstract}

PACS: $33.20 . \mathrm{Kf}, 82.53 . \mathrm{Hn}, 82.53 . \mathrm{Uv}$

\section{Introduction}

Despite the early discovery of photochromism $[1,2]$ and continuous research efforts in this field [3-7], photochromic molecular compounds still attract ample attention due to their distinctive properties. These include the ability to change absorption spectra and / or refractive index [8], modulating molecular fluorescence yield [9] and optically inducing structural changes in supramolecular complexes [10-12]. All these modifications are reversible via thermal back conversion or upon exposure to a light of different wavelength. Essential mechanisms responsible for these photoinitiated changes in photochromic compounds are: transformation in isomerization, intramolecular proton transfer, chemical bond cleavage, or other stereoelectronic modifications [13]. Due to unimolecular character of photochromic transformation, these compounds were proposed to be used for high-density optical data storage, or molecular-scale optoelectronic devices [14].

One of the newly synthesized [15-17] groups of photochromic compounds are indolo[2,1- $b][1,3]$ benzoxazines. These photochromes undergo a fast $\mathrm{C}-\mathrm{O}$ bond cleavage upon UV excitation which results in the formation of two distinct chromophoric groups $[18,19]$.
A broken $\mathrm{C}-\mathrm{O}$ bond causes a formation of $\pi$-bond between nitrogen and chiral carbon atom, thus extending the conjugated system of $3 \mathrm{H}$-indolium. Additionally, the broken bond enables the formation of 4-nitrophenolate anion, which is able to absorb visible light at ca. $430 \mathrm{~nm}$ [16]. These photochromes revert thermally back to their initial state within ten-to-few hundred nanoseconds ${ }^{1}$ after the excitation, and $\mathrm{C}-\mathrm{O}$ bond re-forms [20]. This "on-off" switching time is one of the fastest among the photochromic compounds. Another noticeable feature of these photochroms is an excellent fatigue resistance, which allows thousands of switching cycles with no apparent sign of degradation [20].

In this paper, we present a study on a compound with the simplest chemical structure from the group of indolo[2,1-b][1,3]benzoxazines (IB1 in Fig. 1). Recently it has been shown $[18,20]$ that absorption spectra of IB1 in its opened (IB1b) and closed (IB1a) ring configurations can be sufficiently well understood as a combination of spectral features characteristic of its constituent moieties. Thus, in our transient absorption

\footnotetext{
${ }^{1}$ Note that presented lifetimes are for photochromes in solution; once these molecules are incorporated in polymer matrices, backswitching durations increase by 3-4 orders of magnitude.
} 

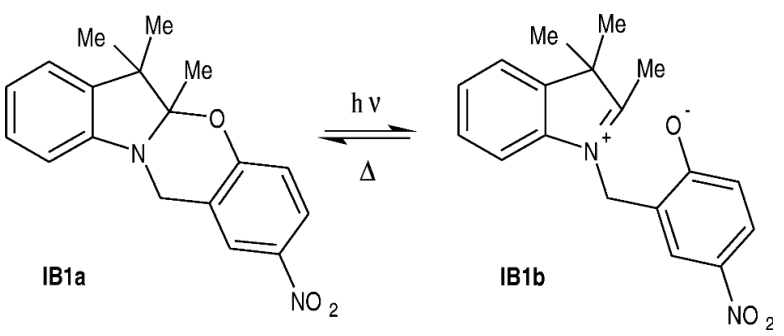

Fig. 1. UV-induced reversible interconversion of indolo[2,1-b] $[1,3]$ benzoxazine IB1a to zwitterion IB1b.

studies, we compare ultrafast responses of IB1 with those of its model compounds, representing two chromophoric groups comprising the photoswitch. To our knowledge, this is the first attempt to investigate ultrafast dynamics in the family of indolo[2,1-b][1,3]benzoxazine photochromes.

\section{Materials and methods}

Sample preparation. Acetonitrile $(\mathrm{MeCN})$ was purchased from Sigma-Aldrich (gradient grade) and used as received. 1,2,3,3-tetramethyl-3H-indolium iodide (IndI in Fig. 2) was synthesized by alkylation of 2,3,3trimethyl- $3 H$-indole with iodomethane and recrystallized from ethanol. IndI solution in $\mathrm{MeCN}$ was used as a model system for $3 H$-indolium cation of IB $1 b$. The concentration was adjusted to have optical density of ca. 0.8 of the sample at the excitation wavelength (Fig. 2). 4-nitrophenol was also purchased from Sigma-Aldrich and, in order to prepare sodium 4-nitrophenolate solution in $\mathrm{MeCN}$, powdered 4-nitrophenol was dissolved in $\mathrm{MeCN}$ (pNph, Fig. 2) and dry sodium carbonate was added to the solution to deprotonate the phenolic hydroxyl group of $\mathrm{pNph}$. After the filtration of precipitates, this yielded a solution of chromophore possessing sodium 4-nitrophenolate (pNphe in Fig. 2). $\mathrm{pNph}$ and $\mathrm{pNphe}$ were used as model systems for closed (IB1a) and opened (IB1b) forms of IB1 nitrophenol moiety [20]. The concentrations of $\mathrm{pNph}$ and pNphe solutions, used in our experiments, were 0.5 and $0.25 \mathrm{mM}$ respectively. Photochromic compound IB1a (5a,6,6-trimethyl-2-nitro-5a,6-dihydro-12H-indo$\mathrm{lo}[2,1-b][1,3]$ benzoxazine) was synthesized according to the procedure described earlier [15]. Solutions of IB1a in MeCN with the corresponding concentrations of $\sim 0.8$ and $1.9 \mathrm{mM}$ were used respectively for fs pump-probe and ns flash-photolysis measurements. All the spectroscopic measurements presented in this study were carried out at room temperature, with the samples stored in closed fused silica cells of $1 \mathrm{~mm}$ pathlength.

Steady-state spectra. Steady-state absorption spec-

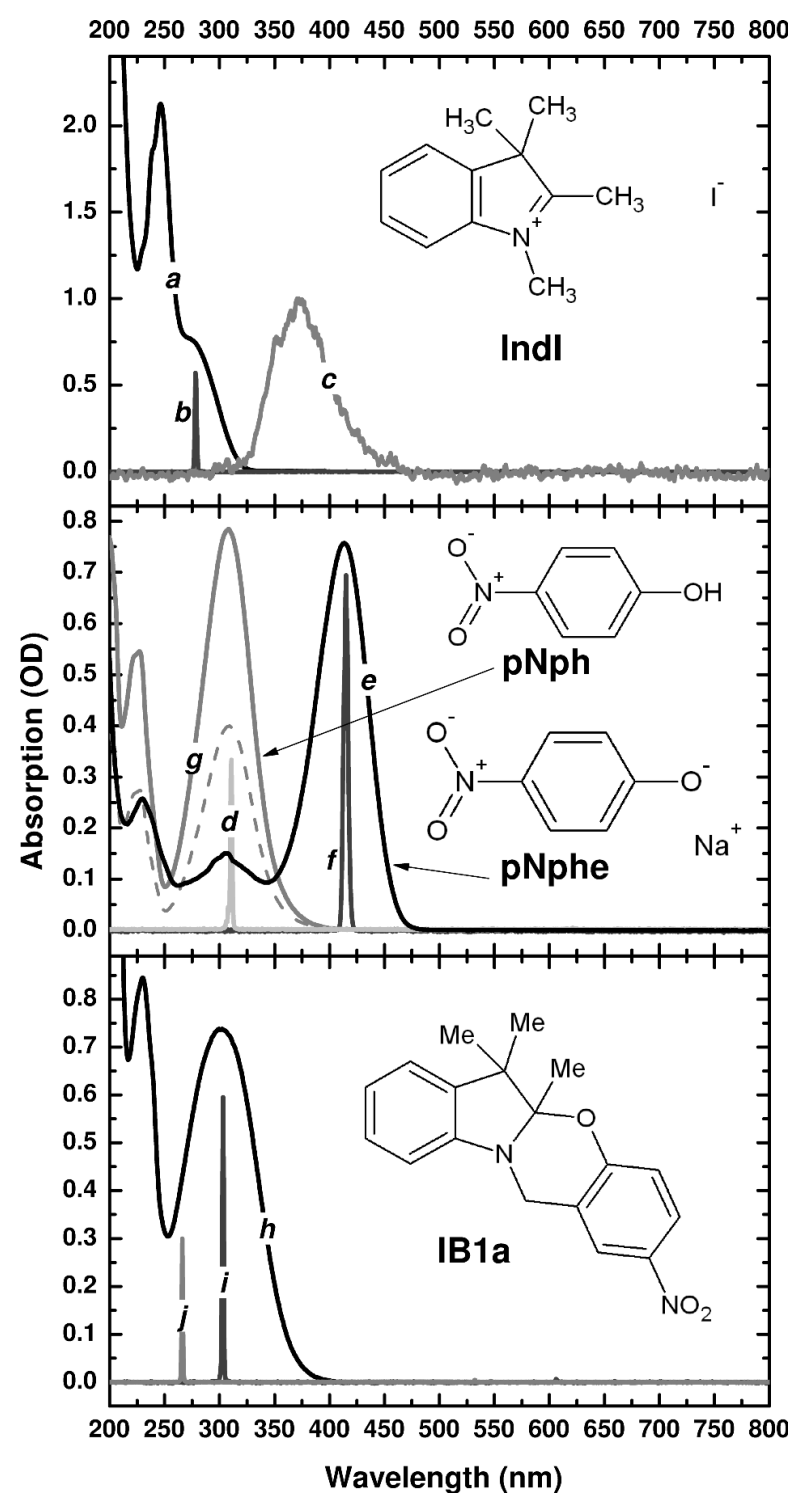

Fig. 2. Steady-state absorption and emission spectra and structural formulae of investigated compounds. Top graph: absorption $(a)$, fs excitation pulse $(b)$, and fluorescence $(c)$ spectra of 2,3,3trimethyl- $3 H$-indolium iodide (IndI) dissolved in MeCN. Middle graph: steady-state sample absorption spectra of 4-nitrophenol (pNph, $g$ ) and sodium 4-nitrophenolate (pNphe, $e$ ), the wavelengths of pump light used in pump-probe experiments on $\mathrm{pNph}$ and $\mathrm{pN}$ phe respectively ( $d$ and $f$ ). Dashed line is pNph absorption with the concentration identical to that of pNphe sample. Lower graph: steady-state absorption spectra $(h)$ and structural formula of photochromic compound IB1a. The excitation laser wavelengths for fs pump-probe $(i)$ and ns flash-photolysis $(j)$ experiments are displayed.

tra were measured using a scanning spectrophotometer (Shimadzu UV-3101PC). Fluorescence spectra were measured by exciting sample with a laser radiation and collecting the emission light into a fibre-optic spectrometer (Avantes AvaSpec-2048).

Pump-probe measurements. For the pump-probe 
measurements we have used a home-built transient absorption spectrometer backed by the femtosecond laser system (Spectra-Physics, Spitfire), which delivered $1 \mathrm{~mJ}$ pulses with the duration of $130 \mathrm{fs}$ at $1 \mathrm{kHz}$ repetition rate. The wavelength of generated radiation was centred at $805 \mathrm{~nm}$, the spectral bandwidth was $\sim 150 \mathrm{~cm}^{-1}$. Optical parametric amplifier (OPA) (TOPAS, Light Conversion) was used to obtain tunable pump pulses matching the sample absorption spectrum. White-light continuum, generated by focusing a weak $805 \mathrm{~nm}$ beam into a $\mathrm{CaF}_{2}$ crystal, was used as a broadband probe. Group velocity dispersion within the probe pulse was characterized by optical Kerr effect in $\mathrm{MeCN}$. The obtained dispersion curve was further refined in the global analysis procedure used to analyse the data. In all figures, the presented data is corrected for the dispersion of the probe light. Temporal delay between the pump and probe pulses was varied by changing the optical path length travelled by the probe pulse that was sent to the hollow retroreflector placed on computer-controlled linear translation stage. Polarizations of pump and probe beams were linear and set to be at the magic angle $\left(54.7^{\circ}\right)$ with respect to each other. Both beams were focused into the sample with the probe diameter set to $\sim 90 \mu \mathrm{m}$ and pump being roughly 2-2.5 times larger. The probe light transmitted through the sample was dispersed using an imaging spectrograph and recorded by linear-array detector (Hamamatsu). Collected spectra had an average noise level of $<0.5 \mathrm{mOD}$, with an exception of 725-800 region where the noise was 2-3 mOD. This increase in the noise was caused by the instabilities of the white-light continuum that were becoming more pronounced in the spectral proximity of the driving $805 \mathrm{~nm}$ pulse. Within one experimental time point, ca. 5000 white light spectra were averaged and difference absorption spectrum was calculated. A typical dataset contained 512 spectral points and ca. 120 time points. Steady-state absorption spectra collected before and after each experiment did not show any substantial signs of photo-degradation.

Flash-photolysis measurements. In the flash-photolysis experiments the sample was excited by exposing it to the 4th harmonic of nanosecond Nd: YAG laser (Ekspla NL301). The pulse energy and excitation wavelength was respectively $6 \mathrm{~mJ}$ and $266 \mathrm{~nm}$. Samples were exposed to an unfocused laser beam with the diameter of ca. $2.6 \mathrm{~mm}$. Light flashes with the duration of $\sim 100 \mu$ s produced by the laser-synchronized Xe flash lamp were used to strobe the sample transmission, whilst temporal changes in its absorption spectra were detected using a monochromator, behind which a high- speed photodiode was placed and connected to a $1 \mathrm{GHz}$ bandwidth oscilloscope (Tektronix TDS 7104). Temporal resolution of these measurements was limited by the duration of our laser pulse and was approximately 4-5 ns. All the nanosecond kinetic traces presented here are averaged from 20 single shot measurements.

Data analysis procedures. The collected time-resolved spectra and traces were analysed using global analysis techniques described before [21,22]. In short, a certain discrete number of compartments, connected inbetween by linear rate kinetics, are assumed as a model system for the data to be fitted. The excitation populates one or several of these compartments and, as time passes, compartment populations are redistributed according to the assumed connectivity scheme. Free parameters of the fit are rates of redistribution between particular compartments and spectra assigned to a certain compartment. For simplicity, data presented in this paper is fitted using a sequential (evolutionary) model, where different compartments are characterized by time-dependent concentrations $c_{i}(t)$ and evolve in series $\left(c_{1}(t) \rightarrow c_{2}(t) \rightarrow \ldots\right)$. These concentrations are described by the following system of differential equations:

$$
\begin{aligned}
\frac{\mathrm{d}}{\mathrm{d} x} c_{1}(t) & =I(t)-\frac{1}{\tau_{1}} c_{1}(t), \\
\frac{\mathrm{d}}{\mathrm{d} x} c_{i}(t) & =\frac{1}{\tau_{i-1}} c_{i-1}(t)-\frac{1}{\tau_{i}} c_{i}(t), \quad i \neq 1,
\end{aligned}
$$

and the model function used to fit the data is

$$
F(t, \lambda)=\sum_{i=1}^{n} c_{i}(t) \sigma_{i}(\lambda)
$$

Here $\tau_{i}$ is the lifetime of $i$ th compartment (a decay rate with which it transforms to the $i+1$ one), $I(t)$ is the input of the system, which populates only the first compartment and has a Gaussian profile with FWHM equal to the instrument response function (IRF), and $\sigma_{i}(\lambda)$ is a species associated difference spectrum (SADS) of $i$ th compartment. The number of compartments $n$ was set to the smallest one which yielded a fit with all the features of spectral dynamics present in collected data. This specific way of fitting should not be seen as an implication that our measured data results from the system governed by a sequential dynamics, but rather it should be regarded as a convenient way of parametrizing and presenting the vast amount of experimental data. 


\section{Results}

\subsection{Steady-state absorption and fluorescence spectra}

Absorption and fluorescence spectra of the investigated compounds together with their structural formulae are presented in Fig. 2. The top part of the figure depicts absorption $(a)$ and emission $(c)$ spectra of IndI. Two overlapping bands are observed in the absorption spectrum: a relatively weak one with the maximum at ca. $278 \mathrm{~nm}$ and another one, almost 3 times stronger, peaking around $247 \mathrm{~nm}$. Photostability tests using the pump light of the pump-probe experiments have revealed that these bands are quite distinct in their photodynamics: an excitation of IndI at $247 \mathrm{~nm}$ causes some 2 to 3 orders of magnitude faster sample degradation while comparing it with a photoexcitation of $278 \mathrm{~nm}$ band in an optically matched IndI sample. Moreover, the position of this band corresponds to the absorption of iodine anion and is due to its charge-transfer-tosolvent band $\left({ }^{2} \mathrm{P}_{3 / 2}\right)$ in $\mathrm{MeCN}[23,24]$. The red edge of this band in MeCN is ca. $270 \mathrm{~nm}$, thus the absorption of the lowest lying band $(278 \mathrm{~nm})$ can be attributed only to $3 \mathrm{H}$-indolium cation. Only this band was excited while conducting our experiments (see the spectrum of excitation pulse in Fig. 2, top panel). The fluorescence spectrum of $3 H$-indolium peaks around $375 \mathrm{~nm}$, which corresponds to a Stokes shift of $9300 \mathrm{~cm}^{-1}$.

Curves $g$ and $e$ in Fig. 2 depict the absorption spectra of $\mathrm{pNph}$ and $\mathrm{pNphe.} \mathrm{Evidently,} \mathrm{the} \mathrm{deprotonation}$ of $\mathrm{pNph}$ and the formation of $\mathrm{pNphe}$ produces a significant red shift in its absorption spectrum: the peak of red-most band shifts from 309 to $415 \mathrm{~nm}$, which corresponds to the $3250 \mathrm{~cm}^{-1}$ decrease in the excitation energy. From the comparison of the spectra of these two species at equal concentrations (dashed line and $e$ line in Fig. 2) it is clear that the twofold increase in a maximum extinction coefficient is associated with aforementioned deprotonation reaction of $\mathrm{pNph}$.

Absorption spectrum of IB1a is dominated by two distinct bands at 230 and $302 \mathrm{~nm}$. The $302 \mathrm{~nm}$ band qualitatively coincides with $\mathrm{pNph}$ absorption. Indeed, it is usually assumed in the literature that the conjugated systems of two chromophoric groups of IB1a molecule do not interact strongly [20]. The pNph absorption maximum is at $309 \mathrm{~nm}$ ( $g$, Fig. 2 and [20]) and its extinction coefficient is $\sim 10 \mathrm{mM}^{-1} \mathrm{~cm}^{-1}$. Meanwhile, the reported values of these numbers for Indoline are $\lambda_{\max } \sim 290 \mathrm{~nm}$ and $\varepsilon \sim 2 \mathrm{mM}^{-1} \mathrm{~cm}^{-1}$ [20,25]. Obviously, the $302 \mathrm{~nm}$ absorption band of IB1a is dominated by $\mathrm{pNph}$ moiety with some minor influence of Indoline part.

\subsection{Pump-probe on $3 H$-indolium iodide (IndI)}

For IndI pumping we have used a $278 \mathrm{~nm}$ OPA output with the bandwidth of $230 \mathrm{~cm}^{-1}$ (Fig. 2 b). Pump pulse energy was $\sim 500 \mathrm{~nJ}$ and estimated instrument response function (IRF), for this configuration, was around 280 fs. Representative pump-probe traces and spectra are displayed in Fig. 3. From the spectra shown in Fig. 3 it is evident that femtosecond dynamics of IndI is dominated by two induced absorption bands which are formed within sub-IRF time scale. These two bands exhibit different behaviour: the first band appears at $337 \mathrm{~nm}$ and within its decay time of ca. 10 ps (Fig. 3, open squares) gradually shifts by $12 \mathrm{~nm}$ to the blue, whilst the second one appears at $580 \mathrm{~nm}$, rapidly (within 200-500 fs) shifts to ca. $528 \mathrm{~nm}$, and then steadily vanishes. The shifting of second band can also be observed in the kinetic traces; $500 \mathrm{~nm}$ trace gradually increases within $500 \mathrm{fs}$ after excitation, while other traces follow more or less instrument response limited growth. In the transient absorption spectra measured on picosecond time scales, broad induced absorption (IA) plateau is observed stretching over the region of $400-800 \mathrm{~nm}$, with an additional IA band peaking at around $725 \mathrm{~nm}$. At times after ca. 2 ps, a negative band appears at ca. $365 \mathrm{~nm}$. Its position roughly corresponds to the peak of IndI fluorescence spectrum (curve $c$ in Fig. 2), indicating that this band can be attributed to stimulated emission (SE) of IndI. Note that this band overlaps with IA plateau and this can cause additional distortion or small conceivable shift.

\subsection{Pump-probe on 4-nitrophenol and 4-nitrophenolate (pNph \& pNphe)}

Excitation of pNph was performed using $311 \mathrm{~nm}$ ( $\sim 170 \mathrm{~cm}^{-1}$ bandwidth) wavelength and $500 \mathrm{~nJ}$ energy fs laser pulses with an estimated IRF of $215 \mathrm{fs}$. Selected pump-probe data are presented in the upper part of Fig. 4. Here, in the initial spectrum, corresponding to 125 fs probe delay, a sharp negative peak is observed at around $342 \mathrm{~nm}$. Its wavelength corresponds to the Stokes shifted pump wavelength (the strongest vibrational band in acetonitrile lies at $2940.8 \mathrm{~cm}^{-1}$ [26]), which, together with the fact that the band is only observed during the time-overlap of pump and probe pulses, shows that the band is due to the impulsive Raman scattering of the pump. Another spectral feature, which appears in the first time-gated spectrum and is more pronounced in the consecutive one of $400 \mathrm{fs}$, is a wide induced absorption band in 350$650 \mathrm{~nm}$ range with a pronounced peak around $500 \mathrm{~nm}$. 


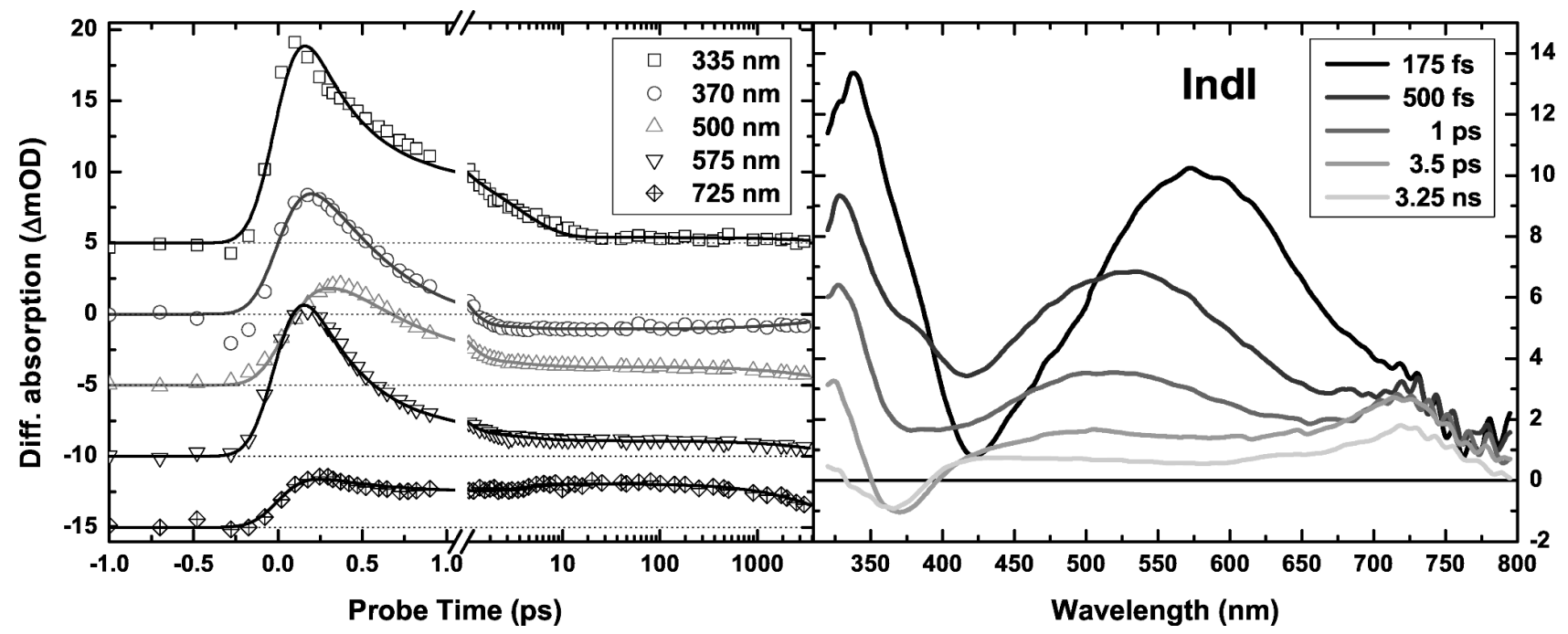

Fig. 3. Left graph: pump-probe traces of IndI for different probe wavelengths (points), with global analysis fit on measured data (solid lines), based on four-compartment sequential model. Experimental and fitted traces were offset vertically to aid the viewing. Doted lines show the real zero-level for appropriate trace. Right graph: time-gated pump-probe spectra measured at various delays (indicated in the legend) after the excitation pulse.

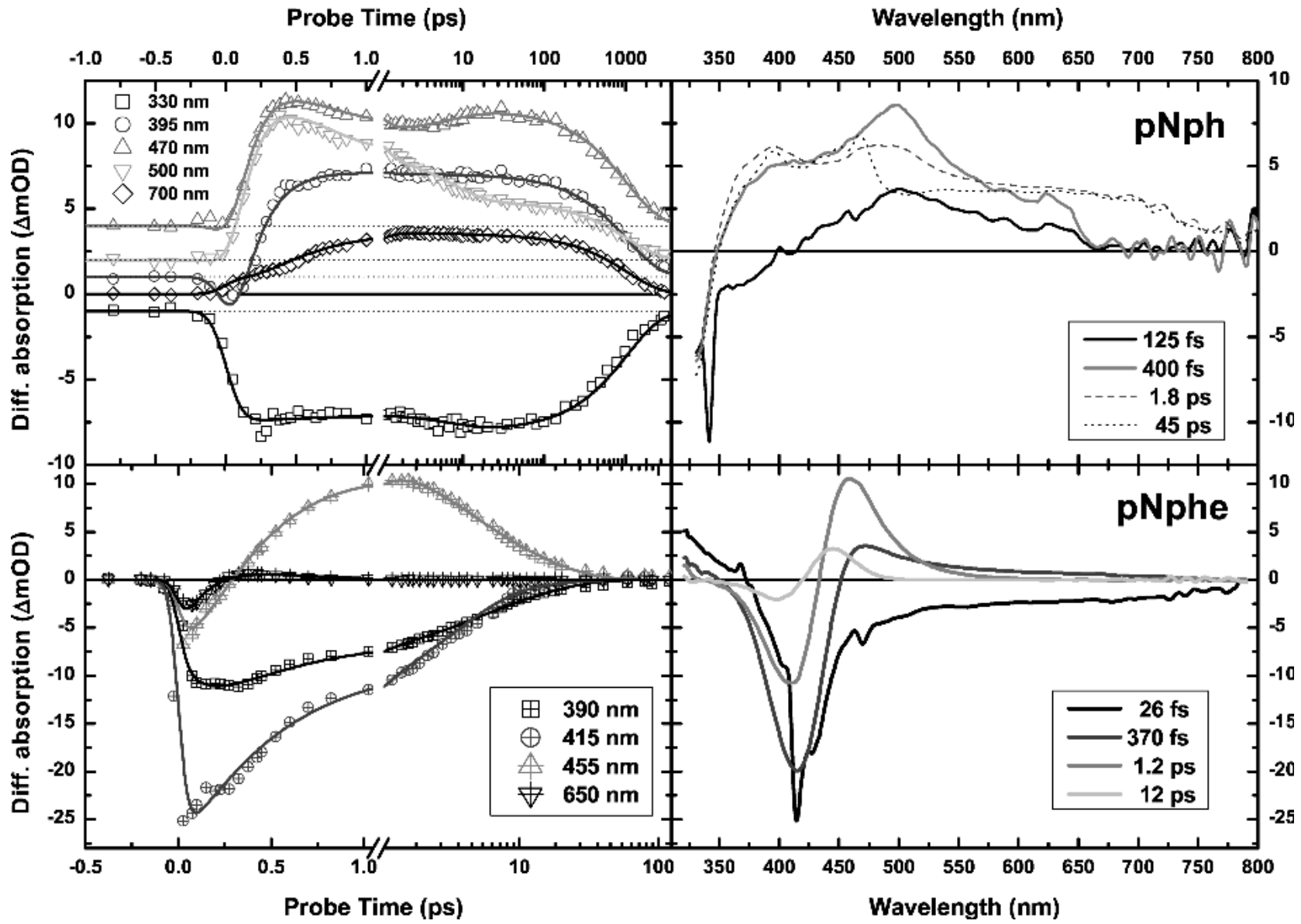

Fig. 4. Selected kinetic traces (left-hand side) and time-gated spectra (right-hand side) from pump-probe experiments on 4-nitrophenol (pNph, upper graphs) and 4-pitrophenolate (pNphe, lower graphs) chromophores. Solid lines show the fit curves obtained using four-compartment sequential model. For clarity, all pNph kinetic curves together with the fits were offset by 1 or $2 \mathrm{mOD}$, while doted lines were added to

illustrate the zero-levels. Probe wavelengths for traces and delay times for spectra are illustrated in the corresponding legends. 
The signal below $350 \mathrm{~nm}$ is negative. This decrease in absorbance matches the red edge of steady-state absorption and is due to the ground state bleaching (GSB). On picosecond time scales, IA additionally extends to the red (up to $800 \mathrm{~nm}$ ) and $500 \mathrm{~nm}$ band vanishes within $\sim 12$ ps (open-down-triangle trace in Fig. 4 ). At 45 ps after the excitation, double band structure becomes evident in the spectrum, with peaks around 400 and $470 \mathrm{~nm}$, on top to wide absorption region dominating the visible and near-infrared parts of spectrum. At later times, $\mathrm{pNph}$ has no apparent spectral redistribution and all spectral components simultaneously decay within $\sim 3$ ns. This can be seen clearly in the kinetic traces, where the decay of GSB band (open squares in Fig. 4) indicates the return to the original ground state. pNphe was excited with a fs laser pulses of $415 \mathrm{~nm}$ wavelength and $280 \mathrm{~cm}^{-1}$ bandwidth (curve $f$ in Fig. 2). Pulse energy and the IRF of the experiment were $175 \mathrm{~nJ}$ and 130 fs respectively. Collected pump-probe data are presented in Fig. 4. An initial transient absorption spec- trum (26 fs delay in Fig. 4), shows a bleaching signal heavily affected by coherent artifacts [27]. Specifically, a cross-phase modulation can be observed as a narrow spike around the pump wavelength, and stimulated Raman scattering band (see above) is observed as a negative peak around $470 \mathrm{~nm}$. After the disappearance of coherent phenomena, GSB can be observed clearly centred around $415 \mathrm{~nm}$, and an induced absorption stretching all over the $450-750 \mathrm{~nm}$ region. Within ca. $1 \mathrm{ps}$, this wide induced absorption structure transforms into a relatively narrow band with a peak absorbance around $460 \mathrm{~nm}$ and as time passes it gradually decreases and shifts to the blue. As it is evident from the kinetic traces, transient absorption signal of pNphe comprised of GSB and induced absorption fully disappears within $40 \mathrm{ps}$ after the excitation.

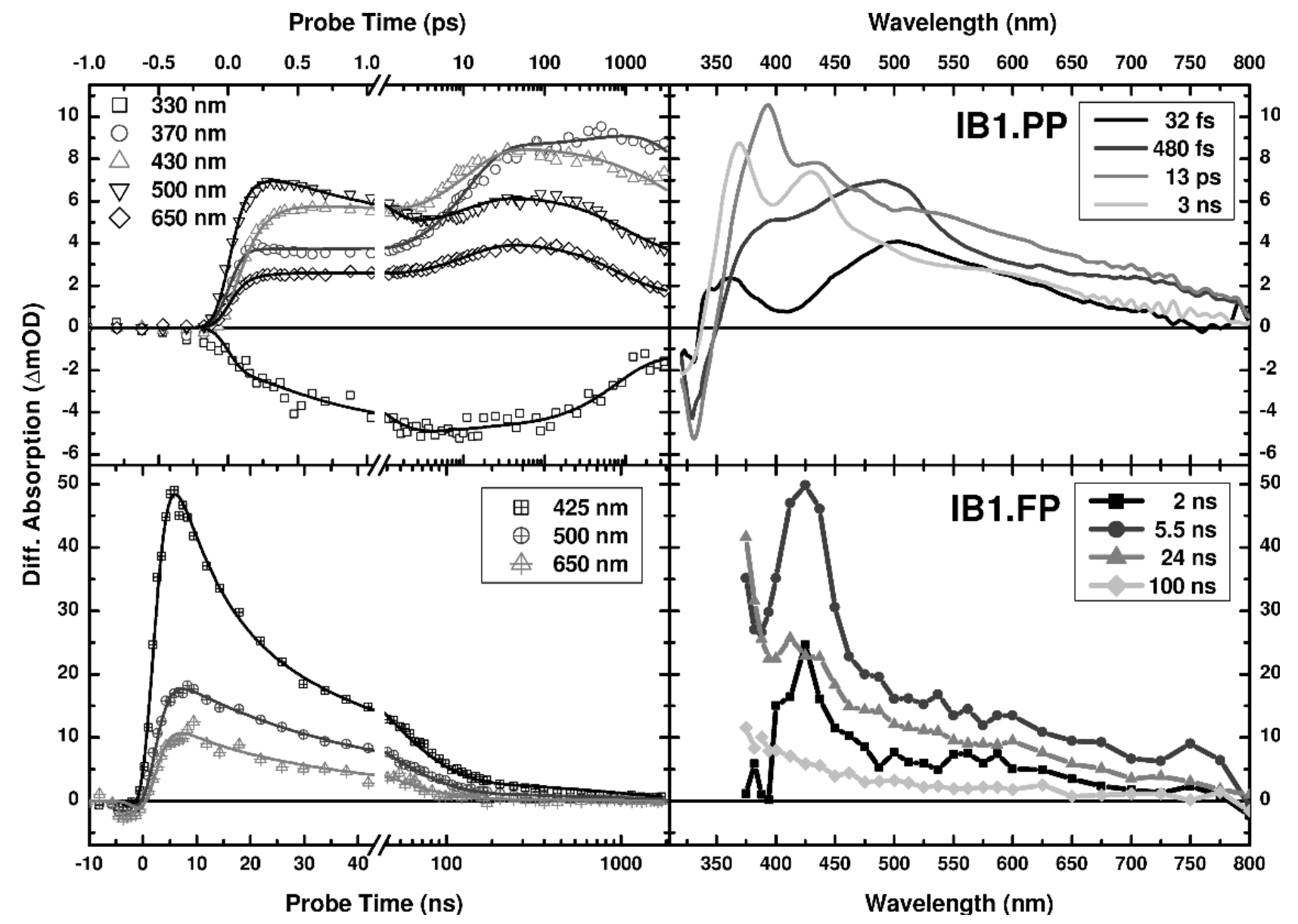

Fig. 5. Results from pump-probe (upper graphs, IB1.PP) and flash-photolysis (lower graphs, IB1.FP) experiments on IB1 photochromic switch. Transient absorption kinetics for characteristic wavelengths (left-hand side graphs) and time-gated spectra for selected delay times (right-hand side graphs). Probe wavelengths and gate times are shown in the legends. Solid lines in the kinetic datasets depict results from global analysis fitting for corresponding probe wavelengths. 


\subsection{Pump-probe and flash-photolysis experiments on IBIa}

Excitation in the pump-probe experiments on photochromic switch IB1 a was performed using the $303 \mathrm{~nm}$ wavelength $\left(180 \mathrm{~cm}^{-1}\right.$ bandwidth, curve $i$ in Fig. 2$)$ and $450 \mathrm{~nJ}$ energy femtosecond pulses. IRF for this configuration was ca. $200 \mathrm{fs}$. Selected kinetic traces and timegated spectra from pump-probe and flash-photolysis experiments on IB1a are presented in Fig. 5 (top). The first time-gated spectrum (32 fs delay in Fig. 5) measured on IB1a shows two absorption bands and GSB signal appearing instantaneously after the excitation. Subsequently, within 500 fs (see 480 fs spectrum in Fig. 5) the signal of GSB increases, while induced absorption bands increase and converge closer to each other. The kinetic traces depict this convergence, or gap disappearance between two absorption bands, by slight slower ingrowth of the transient absorption around $430 \mathrm{~nm}$ (trace of open up-triangles, Fig. 5), compared to that at $500 \mathrm{~nm}$ (down-triangles). Another non-IRF limited change in the kinetics is the increase of GSB signal. This gradual growth is an indication that GSB initially overlaps with an induced absorption band which disappears within ca. 8 ps after the excitation. Dynamic changes occurring on 5-10 ps timescale are present over the entire investigated spectral region; they result in qualitative changes of the shape of transient absorbance spectrum. A timegated spectrum at 13 ps displays a broad absorption plateau in 350-800 $\mathrm{nm}$ region, with two distinct bands on top of it. These bands are centred at 395 and $435 \mathrm{~nm}$. Within subsequent $3 \mathrm{~ns}$, these bands shift respectively by 26 and $5 \mathrm{~nm}$ to the blue, whilst the overall amplitude of absorption plateau decreases by about $40 \%$. Further dynamics, which was observed by the means of flashphotolysis, is not as rich as in the ultrafast time domain (lower graphs in Fig. 5). Here an even more pronounced $430 \mathrm{~nm}$ band is initially observed, placed over the broad induced absorption plateau. This band completely vanishes within 24 ns after the excitation. From this point on, no other spectral features, except the absorption plateau, can be distinguished. The logarithmic part of $425 \mathrm{~nm}$ kinetic trace (Fig. 5, crossed-square) illustrates the decay of absorption plateau, which almost completely disappears in ca. $1 \mu \mathrm{s}$.

\section{Discussion}

The vast amount of presented experimental data and the complexity of transient dynamics in the investigated systems clearly requires a more concise presentation of all the measured data, not just selected plots, to aid the interpretations. Thus we have used global fitting procedures to systematize and compare experimental results (see "Materials and methods", Sec. 2). The analysis results for all investigated compounds, in the form of SADS and their lifetimes, are presented in Fig. 6. The quality of the fit can roughly be judged from the correspondence between fitted (solid lines) and measured kinetic traces shown in Figs. 3-5.

The initial, IRF limited, transient absorption spectrum of our IndI sample (Fig. 3) surprisingly well reproduces the $\mathrm{S}_{1} \rightarrow \mathrm{S}_{n}$ absorption of indole radical cation (Indolyl) in aqueous environment that has been observed by various spectroscopic methods [28-30]. Although indolyl radical and $3 H$-indolium cation investigated here are not exactly the same molecules, the sizes and structures of their conjugated systems are similar, thus presumably leading to a close match between the excited state absorption (ESA) spectra. Another observation that can immediately be made from the spectra is the similarity between the 500 fs time-gated spectrum in Fig. 3 and transient absorption spectrum of $3 \mathrm{H}$ indolium cation in low-pH solution, reported earlier by Jovanovic and Steenken [29]. The fact that the spectral shifts observed on the ultrafast timescales are comparable to those induced by the changes of solvent $\mathrm{pH}$, could indicate that our sample undergoes substantial charge redistribution in the excited state [31]. Alternatively, the observed spectral dynamics could be due to the relaxation among different electronic states in the excited state manifold. In this case, the late spectra (curves 3 and 4 in Fig. 6) would correspond to the relaxed $S_{1}$ state as indicated by the SE band. The stimulated emission observed in the SADS of these compartments is accompanied by a featureless absorption plateau stretching from 425 to $725 \mathrm{~nm}$. This plateau could be attributed to the absorption of dimer anion of $\mathrm{MeCN}$ [32], which is formed upon photoionization of IndI, when the elected electron is trapped by two solvent molecules.

The fitting of $\mathrm{pNph}$ and $\mathrm{pNphe}$ data has allowed attributing the exact timescales to the observed dynamic processes (Fig. 6, bottom). Qualitatively, the spectral relaxation is as described in the results section. Distinct feature of pNph dynamics is the appearance of $500 \mathrm{~nm}$ band absorption, $200 \mathrm{fs}$ past the excitation, and its subsequent decay extending into the second and third fit compartments (pNph, Fig. 6). Another noticeable aspect is the constant amplitude of all SADS in the wavelength region corresponding to GSB. This indicates that all the spectral redistributions prior to the 4th compartment are not associated with the disappearance of exci- 


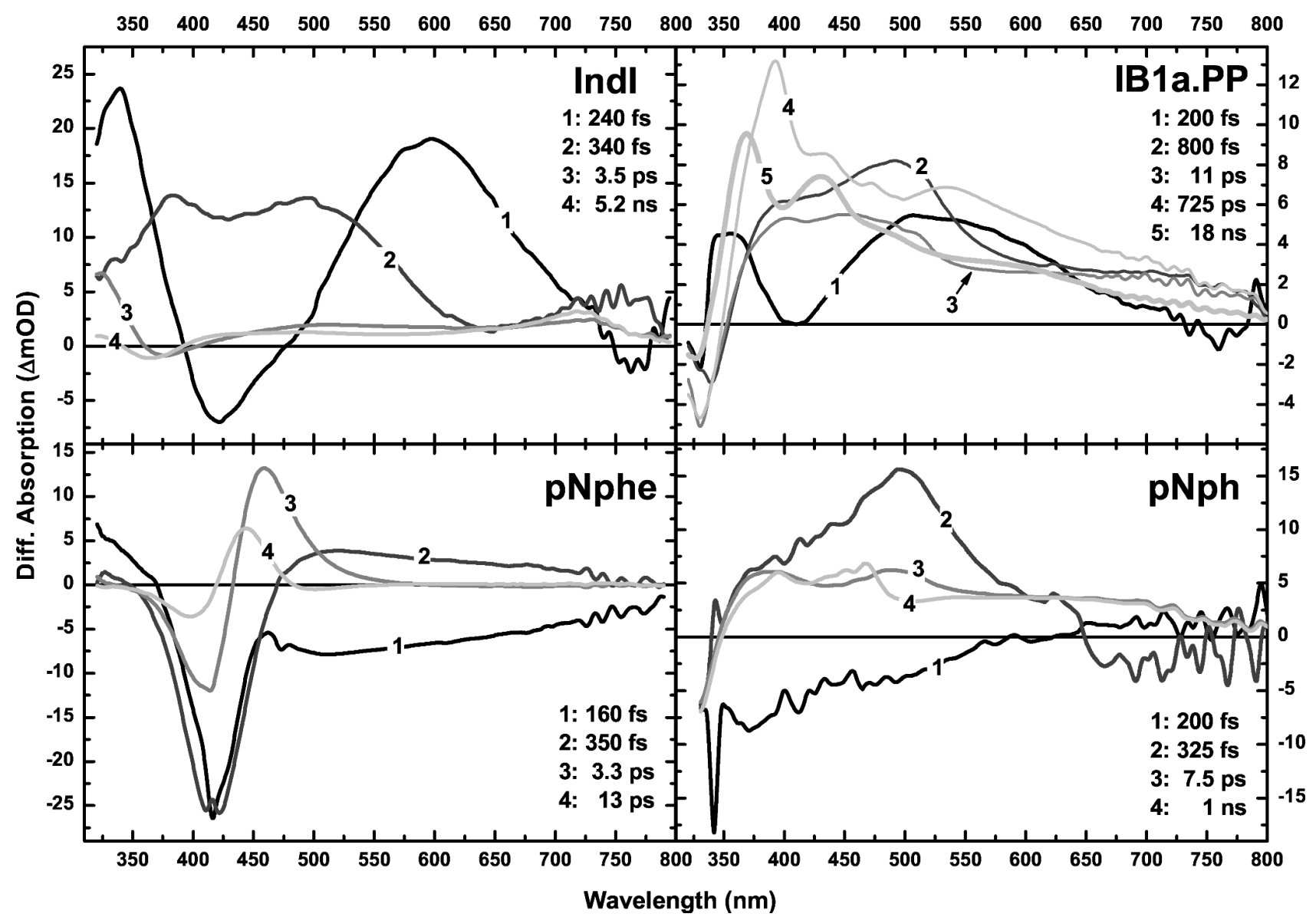

Fig. 6. Estimated compartment species associated difference spectra (SADS) from the global analysis fit on collected pump-probe data for: $3 H$-indolium iodide (IndI), 4-nitrophenoleate (pNphe), photochrom IB1a (IB1a.PP), and 4-nitrophenol (pNph). Sequential dynamics model was assumed for all fits. Compartment's numbers are shown on the spectra and time constants associated with corresponding SADS are given in the legend.

tation, but rather are caused by other phenomena, such as solvation, vibrational relaxation, or internal conversion dynamics within / between the excited state(s). After the redistributions, we see that induced absorption and GSB of the last compartment fully decay with a time constant of $1 \mathrm{~ns}$. Given such a relatively long timescale, we can infer an assumption that this decay is nonradiative, because the emission of this sample could not be detected.

In the SADS of pNphe, we see that GSB band has similar amplitudes only in the first two compartments, thus spectral rearrangement within the excited state takes only $\leq 400$ fs. Afterwards GSB signal continuously decreases while induced absorption shifts to the blue. This fast decrease in GSB could be caused by the presence of conical intersection between reaction surfaces of excited and ground states, whilst the thermalization of "hot" ground state would be consistent with blue shift of induced absorption. Time constant associated with the last SADS of pNphe is $12 \mathrm{ps;}$ comparing it to the corresponding number from $\mathrm{pNph}$ fit, we see that proton removal from 4-nitrophenol increases the excitation relaxation rate by two orders of magnitude. Thus, in the further interpretations the 4-nitrophenolate chromophore could be considered as excitation quencher.

The first eye-catching thing, while comparing SADS of IB1a with the ones of its model compounds, is the similarity between the first compartment spectra of IndI and IB1a.PP. Note that in the comparison of these spectra, one should keep in mind the GSB band present in IB1a, which affects its spectral components below $375 \mathrm{~nm}$; this causes a slight suppression of induced UV absorption band in the first compartment. The spectral resemblance implies that excited $3 H$-indolium cation in IB1 is formed within the IRF of our experiment. This implication is additionally supported by the fact that GSB band in IB1a.PP reaches is maximum at ca. 8 ps after the excitation (open squares in Fig. 5), which closely resembles the overall lifetime of the UV absorption band in IndI (open squares in Fig. 3). None of 
the other model compounds investigated show any substantial induced absorption in $320-340 \mathrm{~nm}$ range. Unfortunately we have not been able to perform a pumpprobe experiment on a compound which would resemble indoline moiety in IB1a (closed ring configuration); but this moiety has a smaller conjugated system, extending only to the benzene ring, thus one would expect that steady-state absorption and, correspondingly, ESA spectra would be blue-shifted with respect to those of IndI. Of course, it is conceivable that abovementioned GSB band suppression can arise from ESA of IB1 molecule as a whole, but it is very unlikely that it would produce analogous transient absorption spectra with a similar decay time as in IndI.

The SADS of IB1a.PP indicate that initial induced absorption, with a double band structure in first compartment, transforms to a broad induced absorbance with pronounced band around $500 \mathrm{~nm}$ (IB1a.PP 2, in Fig. 6). Although excited IndI also produces IA around $500 \mathrm{~nm}$ within $\geq 200$ fs after the excitation, comparison of relative amplitudes in the first and second compartments suggests that apart from further dynamics of excited $3 H$-indolium cation we also see an input from excited nitrophenol ring. Another spectral feature of IB1, which demonstrates dynamics similar to $\mathrm{pNph}$, is a featureless absorption plateau in the red edge of the spectrum above $650 \mathrm{~nm}$. Here constant absorption is observed on subpicosecond and picosecond time scales (compare 2nd and 3rd IB1a.PP compartments with 3rd and 4 th ones of $\mathrm{pNph}$ ). Observation of $\mathrm{pNph-like} \mathrm{dy-}$ namics, at least in the initial steps of IB1a photoexcitation, is consistent with the point that excitation band in steady-state absorption of IB1a is dominated by absorbance from nitrophenol moiety (see description in the results section and [20]).

So far, while discussing the excitation dynamics within the first three compartments of IB1a, we did not encounter any induced absorption peaking around $425 \mathrm{~nm}$. A distinct absorption band around this wavelength would be a sign of nitrophenolate anion formation in IB1b. And indeed, only in the fourth SADS, which is formed at 12 ps after the excitation, we see a minute absorption increase around $425 \mathrm{~nm}$, which in the subsequent compartment transforms to a more pronounced band. This apparently means that nitrophenolate anion in our photochrome forms 12 ps past the excitation, which is in slight contrast to our abovementioned assumption of IRF limited formation of $3 \mathrm{H}$-indolium cation. It may be possible that ring opening in IB1 and formation of two separate ions of zwitterion IB1b do not occur simultaneously. In principle, after the bond cleavage the $3 H$-indolium cation should be formed within the time necessary for the formation of $\mathrm{N}=\mathrm{C}$ bond, a process involving only electronic reconfiguration. In contrast, to produce a stable nitrophenolate anion, IB1 photochrome needs to undergo not only the redistribution of electrons but also experiences a minor conformational change: the distance $\mathrm{C}-\mathrm{O}$ between atoms changes from $1.47 \AA$ in IB1a to $3.07 \AA$ in IB1b (supporting information of [20]). Thus possibly the formations of cation and anion species are associated with different time constants.

Finally, the last spectrotemporal dynamic step of IB1a.PP is the emergence of absorption band around $393 \mathrm{~nm}$ (4th SADS) and its subsequent shift to $369 \mathrm{~nm}$ within 725 ps (5th SADS). Formation of this band is correlated with the appearance of nitrophenolate chromophore and it cannot be ascribed to any of the model compounds investigated. The fact that its appearance is synchronized with the formation of anion in IB1b could mean that it originates from some kind of coherent interaction between anionic part of IB $1 \mathrm{~b}$ and the rest of the molecule. Precondition for this interaction can be found in Fig. 2, where there is an apparent overlap between bands of IndI fluorescence $(c)$ and pNphe absorption $(e)$. Thus the excitation can couple or channel from $3 \mathrm{H}$-indolium cation to nitrophenolate anion, were it would be efficiently quenched.

Tomasulo et al. [20] have recently performed a flashphotolysis experiments on photochrome IB1 and interpreted their results by a simple model: UV induced $\mathrm{C}-\mathrm{O}$ bond cleavage produces a 4-nitrophenolate chromophore which causes an absorption increase in the visible region; subsequently cleaved bond thermally re-forms back and induced absorption vanishes with the time constant of ca. 22 ns. Results of our flashphotolysis measurements on IB1a, in the form of SADS, are presented in Fig. 7. It is obvious that, apart from 4-nitrophenolate anion absorption, there is a longerlived absorption plateau with an increasing absorbance in the blue part of the spectrum (2nd and 3rd compartment in Fig. 7). An absorption band assigned to 4-nitrophenolate is observed only in the first fit compartment, which decays with the lifetime of $8 \mathrm{~ns}$. The following decay of absorption plateau takes about $40 \mathrm{~ns}$ with an exception of UV region where it lingers longer than $1 \mu \mathrm{s}$. The lifetime values of the same compound reported by Tomasulo et al. probably partially incorporate the decay of absorption plateau which has not been clearly resolved in their experiment because of lower signal-to-noise ratio.

From the presented data, it is difficult to find a feasi- 


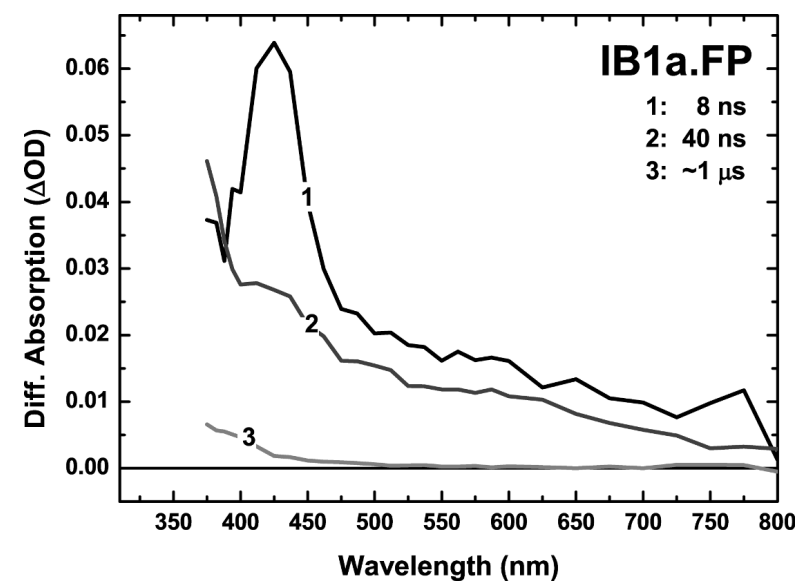

Fig. 7. The SADS resulting from the global analysis fit of photochrom IB1a flash-photolysis data (IB1a.FP). The fit was done assuming three-compartment sequential dynamics model. The associated rate constants are given in the legend.

ble explanation for this featureless induced absorption plateau. It could be caused by some interaction between anionic and cationic moieties of IB $1 b$, although it is strange why it persists by two orders of magnitude longer than 4-nitrophenolate chromophore. On the other hand, this induced absorption could come from a dimer valence anion of $\mathrm{MeCN}[23,32]$ which forms within subpicosecond timescale after the release of electron to solution. However, its spectral shape does not resemble that of the $\mathrm{MeCN}$ dimer anion, and the lifetime of the anion has been reported to be in the millisecond time domain. Yet another possibility for the "long" time component would be the unequilibrated ground state or possible formation of triplet states. Additional experimental work and modelling is necessary to enable better interpretation of this absorption feature. One of the few possibilities would be a search for possible traces of solvated electron in $\mathrm{MeCN}$ which has its absorption maxima around $1.4 \mu \mathrm{m}[23,33]$.

To summarize the results, we propose the following model to synthesize the observations on the ultrafast dynamics in IB1a. UV excitation instantaneously cleaves the $\mathrm{C}-\mathrm{O}$ bond and the excited $3 \mathrm{H}$-indolium cation is formed within $\leq 200$ fs. Subsequently, the molecule undergoes a minor conformational change and 4-nitrophenolate anion is fully formed in $12 \mathrm{ps}$. The following interaction of the two moieties of zwitterion results in the quenching of excitation by the nitrophenolate anion. These dynamic steps probably do not occur with $100 \%$ yields, and shortcuts back to the ground state may be present. One obvious shortcut would be the situation where the conformational change, required for the visible absorbance band to form, fails to occur and the response of 4-nitrophenol moiety instead of 4nitrophenolate is observed.

\section{Conclusions}

We have used a femtosecond pump-probe and nanosecond flash-photolysis techniques to disentangle the excited state dynamics of the indolo[2,1-b][1,3]benzoxazine photochromic switch. The observed forwardswitching dynamics could be partly rationalized by the dynamics of the model compounds, representing different moieties of the photochromic molecule. A scheme of excited state dynamics resulting in photochromism was proposed, that allowed qualitative interpretation of experimental observations. The understanding of the influence of different chromophoric groups in the switching behaviour of this class of photochromes opens the way of intelligent design of the switches with desired spectral and dynamical properties.

\section{References}

[1] E. ter Meer, Über Dinitroverbindungen der Fettreihe, Justus Liebigs Ann. Chem. 181(1), 1-22 (1876).

[2] W. Wislicenus and F. Reitzenstein, Zur Kenntniss des Diketohydrindens, Justus Liebigs Ann. Chem. 277(3), 362-374 (1893).

[3] L. Harris, J. Kaminsky, and R.G. Simard, The absorption spectrum of malachite green leucocyanide and the mechanism of the dark reaction after photolysis, J. Am. Chem. Soc. 57(7), 1151-1154 (1935).

[4] R. Heiligman-Rim, Y. Hirshberg, and E. Fischer, Photochromism in spiropyrans. Part V. On the mechanism of phototransformation, J. Phys. Chem. 66(12), 24702477 (1962).

[5] G.H. Brown, Photochromism (Wiley, New York, 1971).

[6] H. Dürr and H. Bouas-Laurent, Photochromism: Molecules and Systems (Elsevier, Amsterdam, 1990).

[7] J.C. Crano and R.J. Guglielmetti, Organic Photochromic and Thermochromic Compounds (Plenum Press, New York, 1999).

[8] Y.Y. Huang, W. Liang, J.K.S. Poon, Y. Xu, R.K. Lee, and A. Yariv, Spiro-oxazine photochromic fiber optical switch, Appl. Phys. Lett. 88(18), 181102-1-3 (2006).

[9] M. Irie, T. Fukaminato, T. Sasaki, N. Tamai, and T. Kawai, Organic chemistry: A digital fluorescent molecular photoswitch, Nature 420(6917), 759-760 (2002).

[10] I. Willner, Photoswitchable biomaterials: En route to optobioelectronic systems, Acc. Chem. Res. 30(9), 347-356 (1997). 
[11] O. Pieroni, A. Fissi, N. Angelini, and F. Lenci, Photoresponsive polypeptides, Acc. Chem. Res. 34(1), 917 (2001)

[12] T. Hugel, N.B. Holland, A. Cattani, L. Moroder, M. Seitz, and H.E. Gaub, Single-molecule optomechanical cycle, Science 296(5570), 1103-1106 (2002).

[13] H. Bouas-Laurent and H. Durr, Organic photochromism, Pure Appl. Chem. 73(4), 639-665 (2001).

[14] M. Irie, Photochromism: Memories and switches - Introduction, Chem. Rev. 100(5), 1683-1683 (2000).

[15] A.A. Shachkus, Y.A. Degutis, and A.G. Urbonavichyus, Synthesis and study of 5a,6-dihydro12H-indolo[2,1-b][1,3]-benzoxazines, Chem. Heterocyclic Compounds 25(5), 562-565 (1989) [in Russian: Khimiya Geterotsiklicheskikh Soedinenii 25(5), 672-676 (1989)].

[16] M. Tomasulo, S. Sortino, and F.M. Raymo, A fast and stable photochromic switch based on the opening and closing of an oxazine ring, Org. Lett. 7(6), 1109-1112 (2005).

[17] A. Shachkus, J. Degutis, and A. Jezerskaite, 5a,6Dihydro-12H-indolo[2,1-b]-[1,3]-benzoxazines, in: Chemistry of Heterocyclic Compounds, Vol. 35, eds. J. Kovač and P. Zalupsky (Elsevier, Amsterdam, 1988) pp. 518-520.

[18] M. Tomasulo, S. Sortino, and F.M. Raymo, Bichromophoric photochromes based on the opening and closing of a single oxazine ring, J. Org. Chem. 73(1), 118126 (2008).

[19] M. Tomasulo, S. Sortino, and F.M. Raymo, Amplification of the coloration efficiency of photochromic oxazines, Advanced Mater. 20(4), 832-835 (2008).

[20] M. Tomasulo, S. Sortino, A.J.P. White, and F.M. Raymo, Fast and stable photochromic oxazines, J. Org. Chem. 70(20), 8180-8189 (2005).

[21] I.H.M. van Stokkum, D.S. Larsen, and R. van Grondelle, Global and target analysis of time-resolved spectra, Biochim. Biophys. Acta - Bioenergetics 1657(23), 82-104 (2004).

[22] A.R. Holzwarth, Data analysis in time-resolved measurements, in: Biophysical Techniques in Photosynthesis, Vol. 3, eds. J. Amesz and A.J. Hoff (Kluwer Academic, Dordrecht, 1996) pp. 75-92.
[23] C.G. Xia, J. Peon, and B. Kohler, Femtosecond electron ejection in liquid acetonitrile: Evidence for cavity electrons and solvent anions, J. Chem. Phys. 117(19), 8855-8866 (2002).

[24] X.Y. Chen and S.E. Bradforth, The ultrafast dynamics of photodetachment, Annu. Rev. Phys. Chem. 59, 203231(2008).

[25] M.W. Allen, J.R. Unruh, B.D. Slaughter, S.J. Pyszczynski, T.R. Hellwig, T.J. Kamerzell, and C.K. Johnson, Spectroscopy and photophysics of indoline and indoline-2-carboxylic acid, J. Phys. Chem. A 107(30), 5660-5669 (2003).

[26] L.M. Richard, Calibration and validation, in: $R a-$ man Spectroscopy for Chemical Analysis, Vol. 157, ed. J.D. Winefordner (Wiley Interscience, New York, 2000) pp. 251-291.

[27] M. Lorenc, M. Ziolek, R. Naskrecki, J. Karolczak, J. Kubicki, and A. Maciejewski, Artifacts in femtosecond transient absorption spectroscopy, Appl. Phys. B 74(1), 19-27 (2002).

[28] D.V. Bent and E. Hayon, Excited state chemistry of aromatic amino acids and related peptides. III. Tryptophan, J. Am. Chem. Soc. 97(10), 2612-2619 (1975).

[29] S.V. Jovanovic and S. Steenken, Substituent effects on the spectral, acid-base, and redox properties of indolyl radicals - a pulse-radiolysis study, J. Phys. Chem. 96(16), 6674-6679 (1992).

[30] J. Peon, G.C. Hess, J.M.L. Pecourt, T. Yuzawa, and B. Kohler, Ultrafast photoionization dynamics of indole in water, J. Phys. Chem. A 103(14), 2460-2466 (1999).

[31] V. Gulbinas, G. Kodis, S. Jursenas, L. Valkunas, A. Gruodis, J.C. Mialocq, S. Pommeret, and T. Gustavsson, Charge transfer induced excited state twisting of N,N-dimethylaminobenzylidene-1,3-indandione in solution, J. Phys. Chem. A 103(20), 3969-3980 (1999).

[32] Q.K. Timerghazin and G.H. Peslherbe, Electronic structure of the acetonitrile and acetonitrile dimer anions: A topological investigation, J. Phys. Chem. B 112(2), 520-528 (2008).

[33] I.A. Shkrob and M.C. Sauer, Electron localization in liquid acetonitrile, J. Phys. Chem. A 106(39), 91209131 (2002). 


\title{
FOTOCHROMINIO JUNGINIO SU ATSIDARANČIU OKSAZINO ŽIEDU ULTRASPARČIOJI DINAMIKA
}

\author{
M. Barkauskas ${ }^{\text {a }}$, V. Martynaitis ${ }^{b}$, A. Šačkus ${ }^{b}$, R. Rotomskis ${ }^{a}$, V. Sirutkaitis ${ }^{a}$, M. Vengris ${ }^{a}$ \\ ${ }^{a}$ Vilniaus universitetas, Vilnius, Lietuva \\ ${ }^{\mathrm{b}}$ Kauno technologijos universiteto Sintetines chemijos institutas, Kaunas, Lietuva
}

\section{Santrauka}

Atlikti femtosekundiniai žadinimo ir zondavimo bei nanosekundiniai žybsnio fotolizès eksperimentai su naujai susintetintais molekuliniais fotojungikliais. Šiuose fotojungikliuose yra oksazino žiedas, kuris atsidaro molekulei sugèrus UV srities fotoną, o po to per keletą dešimčių nanosekundžiu vèl užsidaro. Tirta žiedo atsidarymo dinamika ir spektrinès fotoproduktų savybės, lyginant laikinès spektroskopijos eksperimentus, atliktus su fotojungikliu, su eksperimentais, atliktais su modeliniais dariniais, atitinkančiais atskiras fotojungiklio grupes. Palyginus gautus rezultatus, pasiūlyta detali molekulinio mechanizmo, lemiančio fotochromiškumą, schema. 